\title{
Peculiar anatomic variation of recurrent laryngeal nerve and EMG change in a patient with right substernal goiter and pre-operative vocal cord palsy-case report
}

\author{
Jia Joanna Wang ${ }^{1}$, I-Cheng Lu $^{2,3}$, Pi-Ying Chang ${ }^{3}$, Che-Wei Wu ${ }^{1,2}$, Ling-Feng Wang ${ }^{1,2}$, Tzu-Yen Huang ${ }^{1,4}$, \\ Feng-Yu Chiang, \\ ${ }^{1}$ Department of Otolaryngology-Head and Neck Surgery, Kaohsiung Medical University Hospital, Kaohsiung Medical University, Kaohsiung, \\ Taiwan; ${ }^{2}$ Faculty of Medicine, College of Medicine, Kaohsiung Medical University, Kaohsiung, Taiwan; ${ }^{3}$ Department of Anesthesiology, Kaohsiung \\ Medical University Hospital, Kaohsiung Medical University, Kaohsiung, Taiwan; ${ }^{4}$ Department of Biological Science and Technology, National \\ Chiao Tung University, Hsinchu, Taiwan; ${ }^{5}$ School of Medicine, College of Medicine, I-Shou University, Kaohsiung, Taiwan; ${ }^{6}$ Department of \\ Otolaryngology, E-Da Hospital, Kaohsiung, Taiwan \\ Correspondence to: Feng-Yu Chiang, MD. Department of Otolaryngology, E-Da Hospital, I-Shou University, No. 1, Yida Road, Yanchao District, \\ Kaohsiung 82445, Taiwan. Email:fychiang@kmu.edu.tw; Tzu-Yen Huang, MD. Department of Otolaryngology-Head and Neck Surgery, Kaohsiung \\ Medical University Hospital, 100 Tzyou First Road, Kaohsiung 807, Taiwan. Email: tyhuang.ent@gmail.com.
}

\begin{abstract}
We report a patient with right substernal goiter and preoperative vocal cord palsy (VCP). During dissection, an anatomic variation of the recurrent laryngeal nerve (RLN) and a prolonged EMG were detected by intraoperative neuromonitoring (IONM). Symmetrical vocal cord movement was recorded one month after surgery. The potential for recovery of vocal cord function is high after surgical treatment of a thyroid tumor with preoperative VCP. An IONM enables early localization of the RLN position. To the best of our knowledge, this case of prolonged EMG in an abnormal acquired-ventral RLN trajectory is the first reported in the literature.
\end{abstract}

Keywords: Preoperative vocal cord palsy; substernal goiter; intraoperative neuromonitoring (IONM); electromyography (EMG); abnormal acquired-ventral recurrent laryngeal nerve (RLN); case report

Submitted Dec 15, 2019. Accepted for publication Mar 06, 2020.

doi: 10.21037 /gs.2020.03.36

View this article at: http://dx.doi.org/10.21037/gs.2020.03.36

\section{Introduction}

Preoperative vocal cord palsy (VCP) is often associated with recurrent laryngeal nerve (RLN) invasion by an advanced thyroid malignancy. However, the reported incidence of preoperative VCP in benign thyroid tumor is $0.2-1 \%(1-3)$. Possible mechanisms of preoperative VCP in benign thyroid lesion include compression, stretching, and inflammation.

Intraoperative neuromonitoring (IONM) is helpful for localizing and identifying the RLN during surgery, for elucidating the mechanism of nerve injury, and for predicting vocal cord function after resection of the thyroid lobe (4). In this case of a right substernal goiter with preoperative $\mathrm{VCP}$, a peculiar anatomic variation of the RLN and an EMG change were detected when IONM was used for early localization of the RLN during lateral thyroid dissection. The RLN was well preserved after surgery, and vocal cord function completely recovered. To the best of our knowledge, this study is the first to report an EMG change in a peculiar anatomic variation of RLN. We present the following case in accordance with the CARE reporting checklist (available at http://dx.doi.org/10.21037/ gs.2020.03.36).

\section{Case presentation}

A 52-year-old male patient without underlying disease suffered from hoarseness for 1 month. Laryngoscopic examination revealed right vocal cord fixation. A computed 


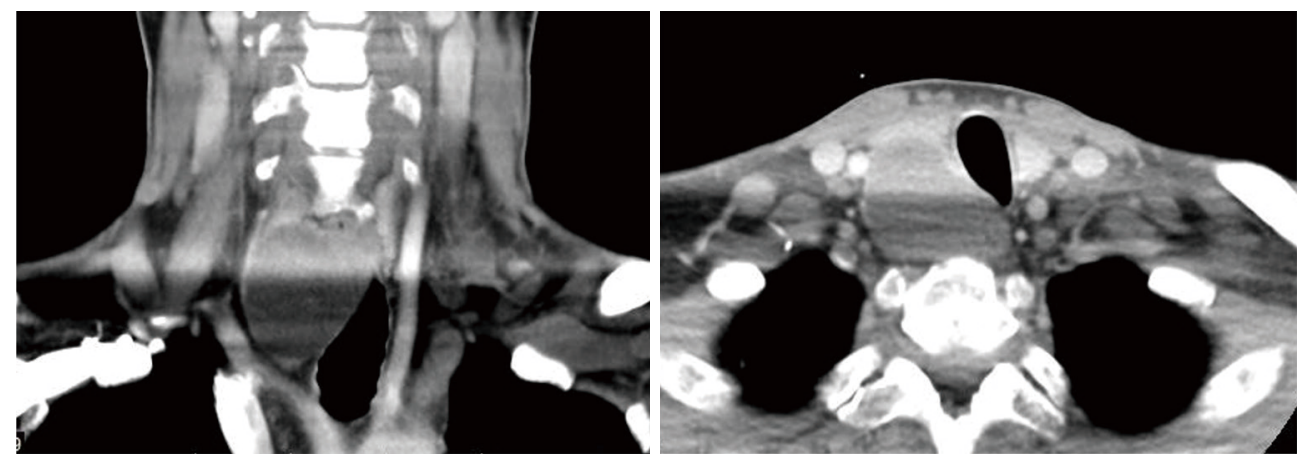

Figure 1 Large right substernal goiter revealed by preoperative computerized tomography scan.
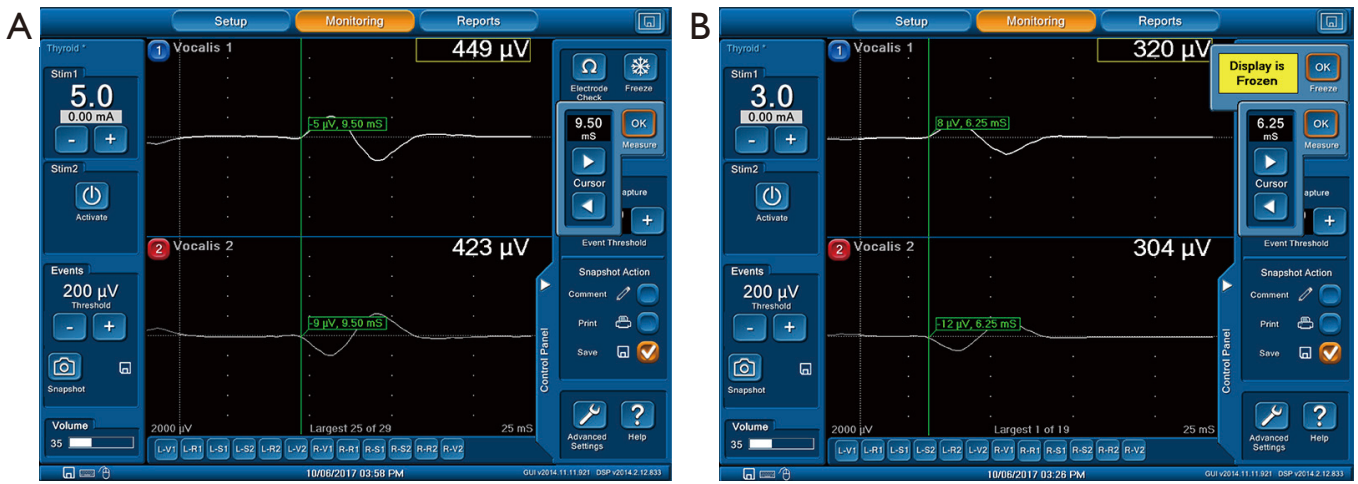

Figure 2 Weak EMG signals with prolonged latency obtained by stimulation (A) vagus nerve for $9.5 \mathrm{mS}$ and (B) recurrent laryngeal nerve for $6.25 \mathrm{mS}$. EMG, electromyography.
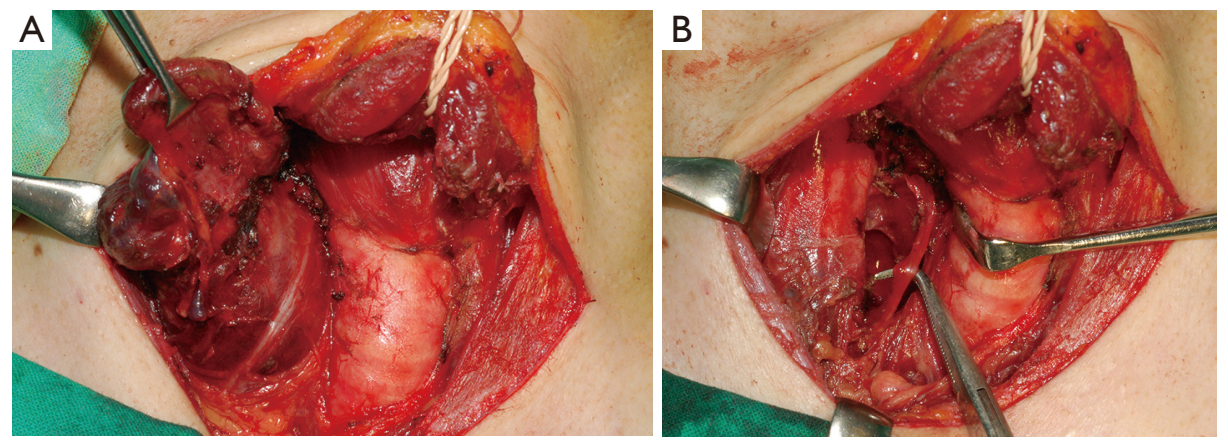

Figure 3 An abnormal acquired-ventral RLN trajectory. (A) The RLN abnormally ran above the thyroid tumor and was compressed by the tumor and clavicle bone; (B) after careful dissection of the RLN, the tumor was completely resected. RLN, recurrent laryngeal nerve.

tomography scan showed a large substernal goiter on the right-side lobe (Figure 1). Thyroid echo and fine needle aspiration cytology indicated a benign thyroid nodule in the Bethesda Reporting System.

During surgery, IONM was performed using the standard procedure (V1-R1-R2-V2) (5). Before thyroid dissection, vagus nerve stimulation revealed a weak EMG signal with prolonged latency of $9.50 \mathrm{mS}$ (Figure 2A). Localization of the RLN revealed the nerve running above the tumor with latency of $6.25 \mathrm{mS}$ (Figure $2 B$ ). The RLN was abnormally positioned on top of the thyroid tumor and was compressed by the tumor and clavicle bone (Figure $3 A$ ). 

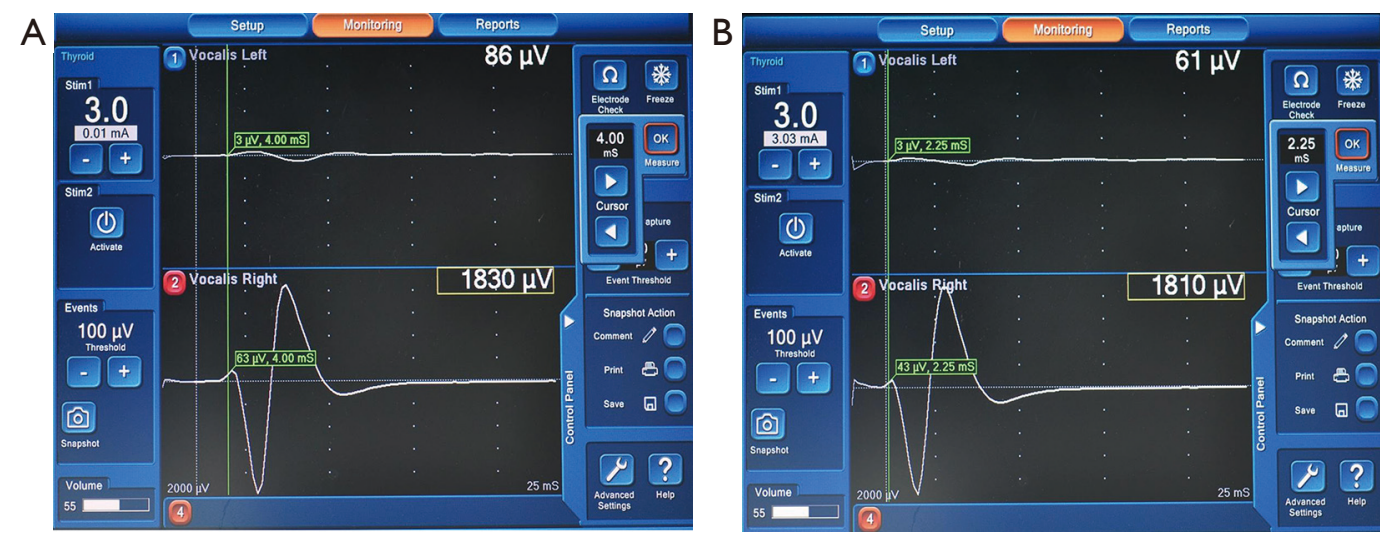

Figure 4 Normal latency after stimulation of (A) right vagus nerve for $4 \mathrm{mS}$ and (B) recurrent laryngeal nerve for $2.25 \mathrm{mS}$.

After careful dissection of the RLN, the tumor was completely resected (Figure 3B). Pathology study supported a diagnosis of benign nodular goiter. One month later, the patient had normal results for subjective voice analysis, and vocal cord movement was symmetrical.

\section{Discussion}

Preoperative RLN palsy may occur in either benign or malignant thyroid tumor. Before unilateral surgery on the lesion side, the RLN must be identified and the neural injury mechanism must be found to ensure selection of an appropriate neural management strategy, including reinnervation. The management and postoperative outcome of the paralyzed RLN will also decisively influence the neural management strategy if contralateral surgery is required. IONM is useful for identifying and preserving the RLN in a thyroid tumor with preoperative VCP. A detectable EMG signal in the case with VCP may indicate residual neural function in the form of retained electrical conductivity. Lorenz et al. reported 41 patients (14\%) out of 285 patients with preoperative VCP had a detectable EMG signal. Among the 41 patients, their VN and RLN amplitudes were significantly lower compared to patients with intact VN and RLN (6). If the RLN is preserved during surgery, functional recovery is as high as $38 \%$ to $89 \%$ in patients whose nerves do not have direct invasion of malignancy $(1,2)$. Therefore, when IONM is performed in patients with preoperative VCP, vagus nerve stimulation should be performed routinely to confirm residual neural function. If an EMG signal is detected, the RLN should be mapped and dissected carefully to confirm that sacrifice of the nerve is unavoidable. In this case, VCP resulted from RLN compression. The EMG signals were consistently weak and had prolonged latency $(9.5 \mathrm{mS}$ latency for right vagus nerve and $6.25 \mathrm{mS}$ latency for RLN). Normal latency for the right vagus nerve and RLN is 4 and $2.25 \mathrm{mS}$, respectively (Figure 4).

In this case, a large goiter with substernal extension protruded from an anatomically abnormal right RLN; instead of ascending in the right tracheoesophageal groove, the right RLN ran across the thyroid tumor. Anatomic variations, including embryological and acquired sources, are classified in the International RLN Anatomic Classification System (7). The patient in this case had an abnormal acquired-ventral RLN trajectory. If IONM is not used for early localization of the RLN, the chance of nerve injury in a patient with an anatomically aberrant nerve is high. During dissection, $3-5 \mathrm{~mA}$ of stimulus current was used to map the full course of the RLN. This report describes this anatomic variant and the importance of early RLN localization (4) during surgery in patients with right substernal goiter. Until now, an anatomic variant of RLN and EMG change has not been described and documented with photographs in the English medical literature.

Worgan et al. reported that a short duration of VCP increases the chance of postoperative recovery of vocal cord function (8). Therefore, thyroid tumor resection and RLN decompression should be performed as soon as possible to maximize the potential for functional recovery of the vocal cords. In this case, surgery was performed 1 month after a VCP diagnosis, and vocal cord function recovered within 1 month after surgery. 


\section{Conclusions}

This case study is a good example of effective surgical treatment in patients with preoperative VCP and demonstrates the following. First, preoperative VCP can occur in benign thyroid tumor. Second, in thyroid tumor with preoperative VCP, surgery should be performed as soon as possible to maximize the potential for recovery of vocal cord function. Third, IONM is still useful for RLN identification and preservation in thyroid tumor patients who have preoperative VCP. Fourth, RLN palsy caused by tumor compression causes a peculiar EMG change and significantly prolonged latency. Finally, for this rare anatomic variant of RLN, early localization of the RLN position should be performed in patients with a right substernal goiter.

\section{Acknowledgments}

Funding: This report was supported by grants from the Kaohsiung Medical University Hospital (KMUH 1077R50, KMUH 108-8M48, KMUH SA10807c), Taiwan.

\section{Footnote}

Conflicts of Interest: All authors have completed the ICMJE uniform disclosure form (available at http://dx.doi. org/10.21037/gs.2020.03.36). CWW reports grants from Kaohsiung Medical University Hospital, Taiwan, during the conduct of the study; TYH reports grants from Kaohsiung Medical University Hospital, Taiwan, during the conduct of the study. The other authors have no conflicts of interest to declare.

Ethical Statement: The authors are accountable for all aspects of the work in ensuring that questions related to the accuracy or integrity of any part of the work are appropriately investigated and resolved. Written informed consent was obtained from the patient for publication of this case report and any accompanying images.

Cite this article as: Wang JJ, Lu IC, Chang PY, Wu CW, Wang LF, Huang TY, Chiang FY. Peculiar anatomic variation of recurrent laryngeal nerve and EMG change in a patient with right substernal goiter and pre-operative vocal cord palsycase report. Gland Surg 2020;9(3):802-805. doi: 10.21037/ gs.2020.03.36
Open Access Statement: This is an Open Access article distributed in accordance with the Creative Commons Attribution-NonCommercial-NoDerivs 4.0 International License (CC BY-NC-ND 4.0), which permits the noncommercial replication and distribution of the article with the strict proviso that no changes or edits are made and the original work is properly cited (including links to both the formal publication through the relevant DOI and the license). See: https://creativecommons.org/licenses/by-nc-nd/4.0/.

\section{References}

1. Holl-Allen RT. Laryngeal nerve paralysis and benign thyroid disease. Arch Otolaryngol 1967;85:335-7.

2. Rowe-Jones JM, Rosswick RP, Leighton SE. Benign thyroid disease and vocal cord palsy. Ann R Coll Surg Engl 1993;75:241-4.

3. Chiang FY, Lin JC, Lee KW, et al. Thyroid tumors with preoperative recurrent laryngeal nerve palsy: clinicopathologic features and treatment outcome. Surgery 2006;140:413-7.

4. Chiang FY, Lu IC, Chen HC, et al. Intraoperative neuromonitoring for early localization and identification of recurrent laryngeal nerve during thyroid surgery. Kaohsiung J Med Sci 2010;26:633-9.

5. Chiang FY, Lee KW, Chen HC, et al. Standardization of intraoperative neuromonitoring of recurrent laryngeal nerve in thyroid operation. World J Surg 2010;34:223-9.

6. Lorenz K, Abuazab M, Sekulla C, et al. Results of intraoperative neuromonitoring in thyroid surgery and preoperative vocal cord paralysis. World J Surg 2014;38:582-91.

7. Randolph GW, Wu CW, Dionigi G, et al. The International RLN Anatomic Classification System. In: Randolph GW, ed. The Recurrent and Superior Laryngeal Nerves. Springer; 2016:125-38.

8. Worgan D, Saunders S, Jones J. Recurrent laryngeal nerve paralysis and the non-malignant thyroid. J Laryngol Otol 1974;88:375-8. 